REVISTA MATEMATICA de la

Universidad Complutense de Madrid.

Volumen 2, nümęro suplementario, 1989

http://dx.doi.org/10.5209/rev_REMA.1989.v2.18096

\title{
On convolution operator in Orlicz spaces
}

\author{
A. KAMIŃSKA and J.MUSIELAK
}

\begin{abstract}
The Orlicz spaces defined on a locally compact abelian group are considered. The main results consist in presenting sufficient and necessary conditions, expressed in terms of gen-

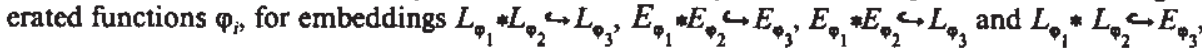
where $L_{\phi}$ are Orlicz spaces and $E_{\phi}$ are their subspaces consisting of all order continuous elements. Some results of the paper are an extension and generalization of those contained in [2], [3], [8] and [10].
\end{abstract}

\section{INTRODUCTION}

The Young theorem ([2], [8]) including sufficient conditions for $L^{p *} L^{q \hookrightarrow} L^{r}(1<p, q, r<\infty)$ has been known for many years. In [8], O'Neil generalizes this result to Orlicz spaces stating sufficient condition for $L_{\varphi}{ }^{*} L_{\varphi} \hookrightarrow L_{\varphi}$. From the other hand there are known sufficient and necessary conditions for the space $L^{p}(I \leqslant p<\infty)$ to be a Banach algebra under convolution as multiplication [10]. A generalization of this result for the Orlicz space is included in [3]. Our main topic consists in finding necessary and sufficient conditions for embeddings of $L_{\phi_{1}} * L_{\phi_{2}}$ into $L_{\phi_{3}}$ and $E_{\phi_{1}} * E_{\phi_{2}}$ into $E_{\phi_{3}}$. We investigate also the other embeddings like $E_{\mathrm{p}_{1}}{ }^{*} E_{\mathrm{p}_{3}}$ into $L_{\mathrm{p}_{3}}, L_{\mathrm{p}_{1}}{ }^{*} E_{\mathrm{p}_{2}}$ into ${ }^{2} L_{\mathrm{p}_{3}}$ and $\mathrm{L}_{\mathrm{p}_{1}}{ }^{*} L_{\mathrm{p}_{2}}$ into $E_{\mathrm{v}_{\mathrm{p}}}$ The Young theorem, the $O$ 'Neil's results and the results concerning the Lebesgue and Orlicz spaces as Banach algebras are obtained as corollaries of our results. In particular we get the necessity of the Young theorem, which seems to be not known so far. We also get the answer to the problem given by $B$. Gramsch in [1].

The first part is devoted to general modular spaces. We give some equivalent conditions in order to a bilinear operator defined on a Cartesian product of modular spaces $X_{p_{1}} \times X_{p_{2}}$ act to another modular space $X_{p_{3}}$. The results of this part are applied to the second one, where the Orlicz spaces and convolution are investigated as modular spaces and the bilinear operator, respectively. The important role is played by conditions $(+)$ and $(++)$ expresing some connections between Young functions $\varphi_{r}$. There are a few versions of those

1980 Mathematics subject Classification (1985 revision): $46 \mathrm{E} 30$

Editorial de la Universidad Complutense. Madrid, 1989 
conditions depending on the kind of a group $G$ and the Haar measure $\mu$. In Theorems 8 and 9 there are given sufficient conditions for $L_{\varphi_{1}} * L_{\varphi_{2}} \hookrightarrow L_{q_{3}}, E_{\mathrm{q}_{1}}{ }^{*}$ $E_{\phi_{2}} \hookrightarrow E_{\varphi_{3}}$ and $L_{\varphi_{1}} * L_{\phi_{2}} \hookrightarrow E_{\phi_{3}}$ by means of the condition $(+)$ and $(++)$. For a discrete group it is posible to prove a converse statement (Theorem 10) without any additional assumptions on the group $G$, whereas for a nondiscrete group the full converse statement (Theorems 11,14 ) is obtained under the assumption of the so called condition $\left(^{*}\right)$ on the group $G$. It is not difficult to check that the groups like $(\mathbb{R},+),(K,+),(T),,(\mathbb{R} \backslash\{0\},),.(K \backslash\{0\}$,$) satisfy condition \left(^{*}\right)$ (Remark 12). At the end there are a number of corollaries including among others, the Young theorem with necessary and sufficient conditions for a large class of locally compact abelian groups.

\section{1.}

Let us now agree on some terminology. Let $\mathbb{R}, \mathbb{K}, \mathbb{N}$ stand for real, complex and natural numbers respectively. Let $X$ be a complex or real vector space. Recall some notions connected with modular spaces [7]. A functional $\rho: X \rightarrow[0,+\infty]$ is called a convex modular if it satisfies the conditions $(1) \rho(0)=0 ;[\forall, \rho(\lambda x)=0] \Rightarrow x=0,(2) \rho\left(e^{i t} x\right)=\rho(x)$ for all $t \in \mathbb{R}(\rho(-x)=\rho(x)$ in the real case), (3) $\rho(\alpha x+\beta y) \leqslant \alpha \rho(x)+\beta \rho(y)$ if $\alpha, \beta \geqslant 0$ and $\alpha+\beta=1$. For any convex modular define the space $X_{p}=\{x \in X: \lim \rho(\lambda x)=0\}=\{x \in X: \rho(\lambda x)<\infty$ for some $\lambda>0\}$ called a modular space and ${ }_{0}^{\lambda \rightarrow 0}=\{x \in X: \rho(\lambda x)<\infty$ for all $\lambda>0\} a$ subspace of $X_{0}$ called the subspace of finite elements. The functional $\|x\|_{\mathrm{p}}=$ inf $\{\varepsilon>0: \rho(x / \varepsilon) \leqslant 1\}, x \in X_{p}$, is a norm in $X_{p}$. The subspace $X_{p}^{X}$ considered with the same norm is closed in $X_{p}$.

1.1. Theorem. Let $\rho_{i}(i=1,2,3)$ be modulars defined on $X$ and $\gamma: X_{\mathrm{p}_{1}} \times X_{\mathrm{p}_{2}} \rightarrow X$ be a bilinear operator. The following conditions are equivalent

(i) For every $\varepsilon>0$ there exists $\delta>0$ such that for all $x \in X_{\mathrm{p}_{1}}, y \in X_{\mathrm{p}_{2}}$ if $\mathrm{p}_{1}(x) \leqslant \delta$ and $\rho_{2}(y) \leqslant \delta$ then $\rho_{3}(\delta \gamma(x, y)) \leqslant \varepsilon$.

(ii) There exists $k_{i}>0(i=1,2,3)$ such that for $x \in X_{\mathrm{p}_{1}}, y \in X_{\mathrm{p}_{2}}$ if $\rho_{1}(x) \leqslant k_{1}$ and $\rho_{2}(y) \leqslant k_{1}$ then $\rho_{3}\left(k_{2} \gamma(x, y)\right) \leqslant k_{3}$.

(iii) There exists $k>0$ such that if $x \in X_{\rho_{1}}, y \in X_{\rho_{2}}$ and $\rho_{1}(x) \leqslant 1$ and $\varepsilon_{2}(y) \leqslant I$ then $\rho_{3}(k \gamma(x, y)) \leqslant 1$.

(iv) There exists $c>0$ such that $\|\gamma(x, y)\|_{\mathrm{\rho}_{3}} \leqslant c\|x\|_{\mathrm{p}_{1}}\|y\|_{\mathrm{p}_{2}}$ for all $x \in X_{\mathrm{p}_{1}}, y \in X_{\mathrm{p}_{2}}$.

(v) $\gamma: X_{\rho_{1}} \times X_{\mathrm{p}_{2}} \rightarrow X_{\mathrm{p}_{3}}$ and $\gamma$ is continuous.

If $\gamma: X_{p_{1}} \times X_{\mathrm{p}_{2}}^{f_{2}} \rightarrow X$ then the analogous conditions to the above in which the spaces $X_{p_{1}}$ are replaced by $X_{p_{i}}^{J}(i=1,2)$ are equivalent. 
Proof. It is enough to give a proof only for $\gamma: X_{p} \times X_{p} \rightarrow X$. The implications $(i) \rightarrow(i i)$ and $(i v) \rightarrow(v)$ are evident and $(i i i) \rightarrow(i v)$ results directly from the definition of a norm in a modular space.

$$
\begin{aligned}
& \text { (ii) } \rightarrow(\text { iii }) \text { if } \rho_{1}(x) \leqslant 1 \text { and } \rho_{2}(y) \leqslant 1 \text { then } \\
& \rho_{1}\left(\min \left(1, k_{1}\right) x\right) \leqslant k_{1} \text { and } \rho_{2}\left(\min \left(1, k_{1}\right) y\right) \leqslant k_{1} \text {, So } \\
& \rho_{3}\left(k_{2} \min ^{2}\left(1, k_{1}\right) \gamma(x, y)\right) \leqslant \max \left(1, k_{3}\right) . \text { Then, by convexity of } \varphi_{3}, \\
& \rho_{3}(k \gamma(x, y)) \leqslant 1 \text { under } k=k_{2} \min ^{2}\left(1, k_{1}\right) / \max \left(1, k_{3}\right) .
\end{aligned}
$$

$(v) \rightarrow(i)$ Suppose $\gamma$ takes its values in $X_{p_{3}}$ and (i) is not satisfied. So there exist $\varepsilon>0$ and sequences $\left(x_{n}\right) \subset X_{p_{1}},\left(y_{n}\right) \subset X_{\rho_{3}}$ such that $\rho_{1}\left(x_{n}\right) \leqslant l / n, \rho_{2}\left(y_{n}\right) \leqslant 1 / n$ and $\rho_{3}\left((1 / n) \gamma\left(x_{n}, y_{n}\right)\right) \geqslant \varepsilon$. Taking $\bar{x}_{n}=(1 / \sqrt{ } n) x_{n}, \bar{y}_{n}=(1 / \sqrt{ } n) y_{n}$ we have $\rho_{1}\left(\lambda \dot{x}_{n}\right) \leqslant \rho_{1}\left(\sqrt{ } n \bar{x}_{n}\right) \leqslant 1 / n \rightarrow 0$ and $\rho_{2}\left(\lambda \bar{y}_{n}\right) \rightarrow 0$, which implies that $\left\|\bar{x}_{n}\right\|_{\rho_{1}} \rightarrow 0$ and $\left\|\bar{y}_{n}\right\|_{\rho_{2}} \rightarrow 0$. However, $\rho_{3}\left(\gamma\left(\bar{x}_{n}, \bar{y}_{n}\right)\right)=\rho_{3}\left((1 / n) \gamma\left(x_{n}, y_{n}\right)\right) \geqslant \varepsilon$. Thus $\left\|\gamma\left(\bar{x}_{n}, \bar{y}_{n}\right)\right\|_{\rho_{3}}^{1}+0$ and $\gamma$ is not continuous.

For some kinds of spaces $X$, modulars $\rho_{i}$ and operators $\gamma$ one can show more.

1.2. Theorem. Let $X$ be a vector lattice. Suppose $\rho_{(}\left(\left|x_{1}\right|\right) \leqslant \rho_{1}\left(\left|x_{2}\right|\right)$ if $\left|x_{1}\right| \leqslant\left|x_{2}\right|$. Moreover, let $X_{p_{1}}$ be complete and a bilinear operator $\gamma: X_{p_{1}} \times X_{p_{2}} \rightarrow X$ be positive, i.e. $\gamma(x, y) \geqslant 0$ if $x \geqslant 0$ and $y \geqslant 0$ and let $|\gamma(x, y)| \leqslant \gamma(|x|,|y|)$.

The following conditions are equivalent

$1 .^{\circ} \gamma: X_{\mathrm{\rho}_{1}} \times \mathrm{X}_{\mathrm{p}_{2}} \rightarrow X_{\mathrm{p}_{3}}$ and $\gamma$ is continuous.

2. $\gamma: X_{\mathrm{p}_{1}} x X_{\mathrm{p}_{2}} \rightarrow X_{\mathrm{p}_{3}}$.

3. There exists $k>0$ such that for $x \in X_{p_{1}}, y \in X_{p_{2}}$ if $\rho_{1}(x) \leqslant 1$ and $\rho_{2}(y) \leqslant 1$ then $\rho_{3}(k \gamma(x, y)) \leqslant l$.

The above conditions in which $X_{\mathrm{p}_{j}}$ are replaced by $X_{p_{f}}^{\mathrm{s}}$ for $i=1,2$, are equivalent, too.

Proof. By the previous theorem only the implication $2 .^{\circ} \rightarrow 3 .^{\circ}$ needs a proof. We shall show it in the case of $X_{p} i=1,2$. For a contrary, let $30^{\circ}$ be not fulfilled. So there exist sequences $\left(x_{n}\right) \subset X_{p_{1}}^{j}, y_{n} \subset X_{p_{2}}^{T}$ such that $\rho_{1}\left(x_{n}\right) \leqslant 1, \rho_{2}\left(y_{n}\right) \leqslant 1$ and $\rho_{3}\left(\left(1 / 2^{2 n} n\right) \gamma\left(x_{n}, y_{n}\right)\right)>1$.

The elements $z=\sum_{n=1}^{\infty} \frac{\mid \underline{x_{n} \mid}}{2^{n}}, w=\sum_{n=1}^{\infty} \frac{\left|y_{n}\right|}{2^{n}}$ belong to $X_{\mathrm{p}_{1}}^{f}, X_{\mathrm{p}_{2}}^{J}$, respectively, 
because of convexity of $\rho_{i}$ and the fact that $X_{p_{i}}^{T}$ are closed subspaces of $X_{p_{i}}$ However,

$$
\begin{gathered}
\rho_{3}((1 / k) \gamma(z, w)) \geqslant \rho_{3}\left(\left(1 / 2^{2 k} k\right) \gamma\left(\left|x_{k},\right| y_{k} \mid\right)\right) \\
\geqslant \rho_{3}\left(\left(1 / 2^{2 k} k\right) \gamma\left(x_{k}, y_{k}\right)\right)>1
\end{gathered}
$$

by assumed properties of $\gamma$. Thus $\gamma(z, w) \notin X_{\rho_{3}}$, which ends the proof.

The results of this section will be applied in the next one to Orlicz spaces and convolution as modular spaces and bilinear operator, respectively.

\section{2.}

Let $\varphi:[0,+\infty] \rightarrow[0,+\infty]$ be convex, left-continuous not identical to zero and infinity, $\varphi(0)=0$ and $\varphi(+\infty)=+\infty$. In the sequel this function will be called a Young function. We say that a Young function is finite if it is finite on $[0,+\infty)$. A generalized inverse function $\varphi^{-1}:[0,+\infty] \rightarrow[0,+\infty]$ is defined as

$$
\varphi^{-1}(y)=\inf \{x \geqslant 0: \varphi(x)>y\} \text {, where inf } \theta=\infty \text {. }
$$

Let $a, b$ be reserved for the following numbers

$$
\begin{aligned}
& a=\sup \{x \geqslant 0: \varphi(x)=0\}, \\
& b=\sup \{x \geqslant 0: \varphi(x)<\infty\} .
\end{aligned}
$$

If a function $\varphi_{i}$ is considered instead of $\varphi$ then $a_{i j} b_{i}$ denote the numbers $a, b$ for the function $\varphi_{i}$.

The connections between $\varphi$ and $\varphi^{-1}$ are formulated in the following simple lemma.

1. Lemma. For all $x \in[0,+\infty]$

$$
x \leqslant \varphi^{-1}(\varphi(x)) \text { and } \varphi\left(\varphi^{-1}(x)\right) \leqslant x .
$$

Moreover,

$$
\begin{gathered}
\varphi\left(\varphi^{-1}(x)\right)=x \text { for } x \in[0, \varphi(b)] \\
\varphi\left(\varphi^{-1}(x)\right)=\varphi(b) \text { for } x \in(\varphi(b), \infty] .
\end{gathered}
$$


We say that two Young functions $\varphi_{1}, \varphi_{2}$ are equivalent for large arguments (small arguments) [all arguments] if

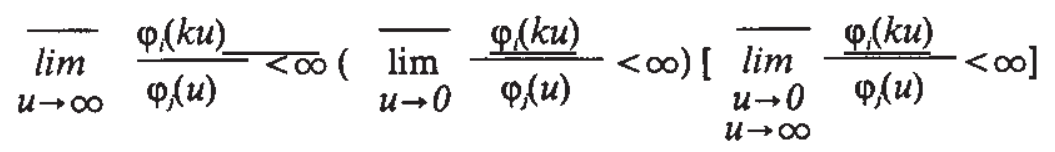

for some $k>0$ and $i, j=1,2$.

In the sequel the expresions "large arguments", "small arguments", "all arguments" will be always denoted by "l.a.", "s.a." and "a.a.", respectively.

In the rest of the paper, $G$ will be a locally compact abelian group, with Haar measure $\mu$. Let $\approx$ the family of all $\mu$-measurable, complex valued functions $f$ defined on $G$. The Orlicz space $L$ is a modular space generated by the modular $I_{0}(f)=\int_{\sigma} \varphi(|f(t)|) d \mu(t)$ defined on $\because$. The subspace of finite elements of the space $L_{\phi}$ is denoted by $E_{q}$. It is well known that, when $\varphi$ is finite, $E_{\text {, consists }}$ of those elements of $L_{\mathrm{o}}$ which are order continuous ([6],[5]). Let us recall that if $\varphi_{1}$ and $\varphi_{2}$ are equivalent for 1.a. (s.a.)[a.a.] then $L_{\varphi_{1}}=L_{t_{2}}$ when $G$ is nondiscrete and compact ( $G$ is nondiscrete and noncompact) [ $G$ is discrete]. If $\varphi_{1}$ and $\varphi_{2}$ are finite then equivalence of these functions implies also that $E_{\varphi_{1}}=E_{q_{2}}$.

In further considerations the important role will be played by the following two conditions. Let $\varphi_{i}, i=1,2,3$, be Young functions.

It is said that $\varphi_{1}$ satisfy condition (+) for l.a.(s.a.)[a.a.] if there exist $k>0$, $\delta>0$ such that

$$
k u v \leqslant \varphi_{1}(u) \varphi_{3}^{-1}\left(\varphi_{2}(v)\right)+\varphi_{2}(v) \varphi_{3}^{-1}\left(\varphi_{1}(u)\right)
$$

when $\varphi_{1}(u) \geqslant \delta$ and $\varphi_{2}(v) \geqslant \delta\left(\varphi_{1}(u) \leqslant \delta\right.$ and $\left.\varphi_{2}(v) \leqslant \delta\right)[u, v \geqslant o]$.

It is said that $\varphi$, satisfy condition (++) for 1.a. (s.a.)[a.a.] if for every $\alpha>0$ there exist $k>0, \delta \geqslant 0$ such that

$$
\alpha u v \leqslant \varphi_{1}(u) \varphi_{3}^{-1}\left(k \varphi_{2}(v)\right)+\varphi_{2}(v) \varphi_{3}^{-1}\left(k \varphi_{1}(u)\right)
$$

when $\varphi_{1}(u) \geqslant \delta$ and $\varphi_{2}(v) \geqslant \delta\left(\varphi_{1}(u) \leqslant \delta\right.$ and $\left.\varphi_{2}(v) \leqslant \delta\right)[u, v \geqslant 0]$.

The above conditions can be reformulated equivalently. Namely, we have the following proposition. The proof will be omitted because it is analogous to that of Lemma 2.4 in [8]. 
2. Proposition.

- Condition (+) for l.a. (s.a.) [a.a.] is equivalent to the following one: there exist $l, \delta>0$ such that

$$
\varphi_{1}^{-1}(u) \varphi_{2}^{-1}(u) \leqslant l u \varphi_{3}^{-1}(u)
$$

if $u \geqslant \delta(u \leqslant \delta)[u \geqslant 0]$.

- Condition (++) for l.a. (s.a.) [a.a.] is equivalent to the following one: for every $a>0$ there exist $l, \delta>0$ such that

$$
\varphi_{1}^{-1}(u) \varphi_{2}^{-1}(u) \leqslant \alpha u \varphi_{3}^{-}(l u)
$$

if $u \geqslant \delta(u \leqslant \delta)[u \geqslant 0]$.

- Condition $(+)$ is invariant under equivalence of the functions $\varphi_{j}$ which is shown in the next proposition.

3. Proposition. If $\varphi_{1}$ satisfy condition (+) for l.a. (s.a.) [a.a.] and $\bar{\varphi}_{i}$ are equivalent to $\varphi_{i}$ for l.a. (s.a.) [a.a.], then $\bar{\varphi}_{i}$ satisfy condition ( + ) for l.a. (s.a.) [a.a.] again.

Proof. For instance, we shall show that $\bar{\varphi}_{i}$ satisfy condition ( + ) for s.a. Since $\vec{\varphi}_{i}$ are equivalent to $\varphi_{i}$, there exist $\delta_{i}, l_{i}>0$ such that $\varphi_{i}\left(l_{i} u\right) \leqslant \bar{\varphi}_{i}(u)$ if $\bar{\varphi}_{i}(u) \leqslant \delta_{i}$ $(\mathrm{i}=1,2)$ and $\bar{\varphi}_{3}\left(l_{3} u\right) \leqslant \varphi_{3}(u)$ if $\varphi_{3}(u) \leqslant \delta_{3}$. Put $l=\min l_{l}$ and $\delta_{o}=\min \delta_{r}$. Without loss of generality suppose $\delta_{o} \leqslant \delta$, where $\delta$ is the constant from condition $(+)$. Then, by condition $(+)$ we have

$k l^{2} u v \leqslant \varphi_{1}(l u) \varphi_{3}^{-1}\left(\varphi_{2}(l v)\right)+\varphi_{2}(l v) \varphi_{3}{ }^{-1}\left(\varphi_{1}(l u)\right) \leqslant \bar{\varphi}_{1}(u) \varphi_{3}{ }^{-1}\left(\bar{\varphi}_{2}(v)\right)+\bar{\varphi}_{2}(v) \varphi_{3}^{-1}\left(\bar{\varphi}_{1}(u)\right)$

if $\bar{\varphi}_{1}(u) \leqslant \delta_{o}$ and $\bar{\varphi}_{2}(v) \leqslant \delta_{\sigma}$

Since $\bar{\varphi}_{3}(l u) \leqslant \varphi_{3}(u)$ when $\varphi_{3}(u) \leqslant \delta_{o^{\prime}}$ so $\varphi_{3}^{-1}(u) \leqslant(l / l) \bar{\varphi}_{3}^{-1}(u)$ if $u \leqslant \delta_{o^{\prime}}$ Indeed, putting $v=\varphi_{3}(u)$ we have $l u \leqslant \bar{\varphi}_{3}^{-1}\left(\bar{\varphi}_{3}(l u)\right) \leqslant \bar{\varphi}_{3}^{-1}(v)$ for $v \leqslant \delta_{o}$. But for $\delta_{o}>v=\varphi_{3}(u)>0$, $u=\varphi_{3}^{-1}\left(\varphi_{3}(u)\right)=\varphi_{3}^{-1}(v)$ and so $\varphi_{3}^{-1}(v) \leqslant(1 / l) \bar{\varphi}_{3}^{-1}(v)$. If $v=\varphi_{3}(u)=0$ then the inequality is also true because $\bar{\phi}_{3}\left(l a_{3}\right) \leqslant \varphi_{3}\left(a_{3}\right)=0$ implies $l a_{3} \leqslant a_{3}$, i.e. $\varphi_{3}^{-1}(0) \leqslant(1 / l) \bar{\varphi}_{3}^{-1}(0)$. Thus

$$
k l^{2} u v \leqslant(l / l) \bar{\varphi}_{1}(u) \bar{\varphi}_{3}^{-1}\left(\bar{\varphi}_{2}(v)\right)+(I / l) \bar{\varphi}_{2}(v) \bar{\varphi}_{3}^{-1}\left(\bar{\varphi}_{1}(u)\right)
$$


If $\bar{\varphi}_{1}(u) \leqslant \delta_{o}$ and $\bar{\varphi}_{2}(v) \leqslant \delta_{o}$ which means that $\bar{\varphi}_{i}$ satisfy ccondition (+) for s.a.

Now we shall discuss the case of a discrete group $G$ in the connection with the Orlicz space and its subspace of finite elements. Traditionally, in this case the notations $l_{*}$ and $h_{\psi}$ are used instead of $L_{*}$ and $E_{*}$. First let us note the following simple fact.

4. Lemma. For every Young function $\varphi$ there exists a Young function $\tilde{\varphi}$ finite and equivalent to $\varphi$ for s.a.

As a corollary, it is seen that instead of $l_{\text {, }}$, where $\varphi$ can take infinite values, one can always consider the isomorphic space $l_{\phi}$, where $\tilde{\varphi}$ is finite. But there are some problems with the subspace of finite elements. If $\varphi$ is infinite for some real numbers, then $h_{\mathrm{q}}=\{0\}$, whereas $h_{\mathrm{q}}$ is always different than $\{0\}$ if $\varphi$ is finite. Thus an equivalent function $\tilde{\phi}$ defines a different subspace of finite elements than the function $\varphi$. However, let us note that for any function $\varphi$ there exists the only subspace of finite elements defined by a function $\bar{\varphi}$ finite and equivalent to $\varphi$. This subspace $h_{\bar{\phi}}$ does not depend on the choice of the function $\bar{\varphi}$, belonging to the class of all Young functions finite and equivalent to $\varphi$.

Taking into considerations the above remarks, in the sequel we shall always assume that $\varphi$ is finite in the case of a discrete group.

The Lemmas 5,6 and 8 are some technical steps to prove Theorems 7 and 9.

5. Lemma. If $\varphi_{i}$ are finite and satisfy condition ( + ) for s.a., then there exist functions $\bar{\varphi}_{i}$ finite and equivalent to $\varphi_{i}$ for s.a. satisfy condition (+) for a.a., if $\varphi_{i}$ satisfy condicion ( +$)$ for l.a., then there exist funtions $\bar{\varphi}_{i}$ equivalent to $\varphi_{i}$ for l.a. and satisfying condition (+) for a.a.

Proof. Let first $\varphi_{i}$ satisfy condition $(+)$ for s.a. Put $h(u, v)=\varphi_{1}(u) \varphi_{3}^{-1}\left(\varphi_{2}(v)\right)+\varphi_{2}(v) \varphi_{3}^{-1}\left(\varphi_{1}(u)\right)$ and $\bar{h}(u, v)$ if $\varphi_{i}$ are replaced by $\bar{\varphi}_{i}$ Let $u_{i}$ be such that $\varphi_{i}\left(u_{i}\right)=\delta$ and put

$$
\varphi_{i}(u)=\left\{\begin{array}{l}
\varphi_{i}(u), \quad u \in\left[0, u_{i}\right] \\
\varphi_{i^{+}}^{\prime}(u) u+\varphi_{i}\left(u_{i}\right)-\varphi_{i^{+}}^{\prime}\left(u_{i}\right) u_{i}, \mathrm{u} \in\left(u_{i}, \infty\right),
\end{array}\right.
$$

where $\varphi_{i+}^{\prime}$ is a right-hand derivative of $\varphi_{i-}$ We have $\varphi_{i}(u) \geqslant \bar{\varphi}_{+}(u)$ and $\varphi_{i}^{-1}(v) \leqslant \bar{\varphi}_{i}^{1}(v)$ for all $u, v \geqslant 0$. We shall show that $\bar{\varphi}_{i}$ satisfy condition $(+)$ for a.a. For $u \leqslant u_{1}, v \leqslant u_{2}$ the inequality is immediate. Let $u \geqslant u_{1}$ and $v \geqslant u_{2}$. Then we can write $\bar{\varphi}_{i}(u)=c_{i} u+d_{i}$ for $u \geqslant u_{i}$, where $c_{i}>0$ and $d_{i}<0$. Hence we simply obtain 


$$
\bar{\varphi}_{1}(u) \bar{\varphi}_{3}^{-1}\left(\bar{\varphi}_{2}(v)\right)=\frac{c_{1} c_{2}}{c_{3}} u v+\frac{c_{1} d_{2}}{c_{3}} u+\frac{d_{1} c_{2}}{c_{3}} v+M,
$$

where $\mathrm{M}$ is a constant dependent on $c_{p} d_{i}$. Since $c_{1} c_{2} / c_{3}>0$, there exist $e_{1}>0$ and $u_{o}>\max \left(u_{1}, u_{2}\right)$ such that

$\bar{\varphi}_{1}(u) \bar{\varphi}_{3}^{-1}\left(\bar{\varphi}_{2}(v)\right) \geqslant e_{1} u v$ whereas $u, v \geqslant u_{\sigma}$. Moreover,

$$
\frac{\bar{\varphi}_{1}(u) \bar{\varphi}_{3}^{-1}\left(\bar{\varphi}_{2}(v)\right)}{u v} \geqslant \frac{\vec{\varphi}_{1}\left(u_{1}\right) \bar{\varphi}_{3}^{-1}\left(\bar{\varphi}_{2}\left(u_{2}\right)\right)}{u_{o}^{2}}=\frac{\delta u_{3}}{u_{o}^{2}}>0
$$

for $u \in\left[u_{1}, u_{o}\right]$ and $v \in\left[u_{2}, u_{o}\right]$.Then for $e=\min \left(e_{1}, \frac{\delta u_{3}}{u_{o}^{2}}\right)$ and $u \geqslant u_{1}, v \geqslant u_{2}$ we have $\bar{h}(u, v) \geqslant e u v$.

Let now $u \geqslant u_{1}$ and $v \leqslant u_{2}$. Then

$$
\bar{h}(u, v) \geqslant\left(\varphi_{3}^{-1}\left(\varphi_{2}(v)\right)+\varphi_{2}(v)\right) \min \left(c_{1} u+d_{1}, \frac{c_{1}}{c_{3}} u+\frac{d_{1}}{c_{3}}-\frac{d_{3}}{c_{3}}\right)
$$

However, by the assumption (+) we have

$k u_{1} v \leqslant \delta \varphi_{3}^{-1}\left(\varphi_{2}(v)\right)+\varphi_{2}(v) u_{3}$ for $v \leqslant u_{2}$. Hence

$\max \left(\varphi_{3}^{-1}\left(\varphi_{2}(v)\right), \varphi_{2}(v)\right) \geqslant e_{2} v$, where

$e_{2}=\min \left((k / 2 \delta) u_{1}, k u_{1} / 2 u_{3}\right)>0$. Therefore

$$
\bar{h}(u, v) \geqslant e_{2} v \min \left(c_{1} u+d_{1}, \frac{c_{1}}{c_{3}} u+\frac{d_{1}}{c_{3}}-\frac{d_{3}}{c_{3}}\right)
$$

for $u \geqslant u_{1}$ and $v \leqslant u_{2}$. Since $\bar{\varphi}_{1}\left(u_{1}\right)=\delta>0$ and $\bar{\varphi}_{3}^{-1}\left(\bar{\varphi}_{1}\left(u_{1}\right)\right)=u_{3}>0$ and the functions $\bar{\varphi}_{i}(u)$ and $\bar{\varphi}_{3}^{-1}\left(\bar{\varphi}_{1}(u)\right)$ are linear for $u \geqslant u_{1}$, there exists a constant $e_{3}>0$ such that

$$
\min \left(c_{1} u+d_{1}, \frac{c_{1}}{c_{3}} u+\frac{d_{1}}{c_{3}}-\frac{d_{3}}{c_{3}}\right) \geqslant e_{3} u \text { for } u \geqslant u_{1} \text {. }
$$

Hence $\bar{h}(u, v) \geqslant e_{2} e_{3} u v$ when $u \geqslant u_{1}$ and $v \geqslant u_{2}$. So we proved the first part of the lemma.

Now let $\varphi_{i}$ satisfy condition ( + ) for l.a. It is not difficult to verify that the functions 


$$
\bar{\varphi}_{i}(u)=\left\{\begin{array}{l}
\varphi_{i}(u) \text { if } \varphi_{i}(u)>\delta \\
\left(\delta / \varphi_{i}^{-1}(\delta)\right) u \text { if } \varphi_{i}(u) \leqslant \delta
\end{array}\right.
$$

satisfy condition $(+)$ for a.a. Moreover, it is evident that they are equivalent to $\varphi_{i}$ for l.a. which finishes the proof of the lemma.

6. Lemma. If $\varphi_{1}$ satisfy condition $(+)$ for a.a. and $I_{\varphi}(g) \leqslant 1$ and $I_{\sigma_{1}}((2 \lambda / k) f) \leqslant 1$ (or $I_{v_{1}}(f) \leqslant 1$ and $I_{\varphi_{2}}((2 \lambda / k) g) \leqslant 1$ where $k$ is the constant from $(+)$, then $I_{q_{3}}\left(\lambda f_{* g}\right)<\infty$.

Proof. Applying (+) we obtain

$$
\begin{gathered}
I_{\varphi_{3}}\left(\lambda f_{*} * g\right) \leqslant \int_{G} \varphi_{2}\left[1 / 2 \int_{G} \varphi_{1}(2 \lambda / k d f(t) \mid) \varphi_{3}^{-1}\left(\varphi_{2}\left|g\left(t^{\cdot} x\right)\right|\right)\right) d \mu \\
\left.\left.+1 / 2 \int_{G} \varphi_{2}\left(g\left(t^{-1} x\right)\right)\right) \varphi_{3}^{-1}\left(\varphi_{1}(2 \lambda / k f f(t))\right) d \mu(t)\right] d \mu(x) \\
\left.\leqslant 1 / 2 \int_{G} \varphi_{3}\left[\int_{G} \varphi_{1}(2 \lambda / k f f(t))\right) \varphi_{3}^{-1}\left(\varphi_{2}\left(\left|g\left(t^{-1} x\right)\right|\right)\right) d \mu(t)\right] d \mu(x) \\
+1 / 2 \int_{G} \varphi_{3}\left[\int_{G} \varphi_{2}\left(\left|g\left(t^{-1} x\right)\right|\right) \varphi_{3}^{-1}\left(\varphi_{1}(2 \lambda / k f f(t))\right) d \mu(t)\right] d \mu(x)
\end{gathered}
$$

Since $I_{\Phi_{1}}(2 \lambda / k f) \leqslant 1$ and $I_{q_{2}}(g) \leqslant 1$, by Jensen's inequality $I_{\varphi_{3}}\left(\lambda f_{* g}\right) \leqslant I_{\omega_{1}}(2 \lambda / k f) I_{\omega_{2}}(g) \leqslant 1<\infty$.

The next theorems give sufficient cconditions for embeddings of the spaces $L_{\boldsymbol{\varphi}_{1}} * L_{\boldsymbol{q}_{2}}, E_{\boldsymbol{\varphi}_{1}} * E_{\boldsymbol{q}_{2}}$ into $L_{\boldsymbol{q}_{3}}, E_{\boldsymbol{\varphi}_{3}}$.

7. Theorem. I. Let $G$ be nondiscrete and $\varphi_{i}$ satisfy condition $(+)$ for l.a. if $G$ is compact and $(+)$ for a.a. if $G$ is noncompact. Then $L_{\varphi_{1}}{ }^{*} L_{\phi_{2}} \rightarrow L_{\varphi_{3}}$. If additionally $\varphi_{3}$ is finite, then $E_{q_{1}}{ }^{*} E_{\varphi_{2}} \hookrightarrow E_{\varphi_{1}}$.

II. Let $G$ be discrete and $\varphi_{1}^{2}$ satisfy condition $(+)$ for s.a. Then $h_{\phi_{1}} * h_{\varphi_{2}} \subset h_{q_{3}}$ and $l_{\varphi_{1}} * l_{\Phi_{2}} \hookrightarrow l_{\phi_{3}}$

Proof. By Theorem 1.2 it is enough to prove only inclusions. I. Let first $G$ be noncompact and $\varphi$ satisfy $(+)$ for a.a. The proof of the inclusion $L_{q}{ }^{*} L_{\varphi} \subset L_{\phi}$ is an immediate consequence of the previous lemma. Really, taking $f \in L_{\text {e }}$ and $g \in L_{\varphi}$ from the unit balls we have $I_{0}(f) \leqslant 1$ and $I_{0}(g) \leqslant 1$. So we can apply the lemma with $\lambda=k / 2$ and thus $I_{\varphi_{3}}\left(k / 2 f_{* g}\right)<\infty$, which means that $f * g \in L_{\rho_{3}}$.

To prove the inclusion $E_{\mathrm{q}}{ }^{*} E_{\mathrm{q},} \subset E_{\mathrm{q},}$ take $f \notin E_{q,}, g \in E_{\mathrm{q},}$. Let $\lambda=3 \beta$, where $\beta$ is arbitrary. Since the Haar measure $\mu$ is regular and $I_{\varphi_{1}}(2 \lambda / k f)<\infty$ and 
$I_{\mathrm{\sigma}_{2}}(2 \lambda / k g)<\infty$, there exist compact sets $G_{1}, G_{2}$ such that $I_{\mathrm{q}_{1}}\left(2 \lambda / k f \chi_{G_{G} G_{1}}\right) \leqslant 1$ and $I_{\sigma_{2}}^{2}\left(2 \lambda / k g \chi_{G G_{2}}\right) \leqslant 1$. We can write

$$
\begin{aligned}
& I_{\oplus_{3}}(\beta f * g) \leqslant 1 / 3 I_{\varphi_{3}}\left(\lambda f \chi_{\sigma G_{1}} * g\right)+ \\
& 1 / 3 I_{\varphi_{3}}\left(\lambda f \chi_{\sigma_{1}} * g \chi_{G_{-G}}\right)+1 / 3 I_{\Phi_{3}}\left(\lambda f \chi_{G_{1}} * g \chi_{G_{2}}\right)
\end{aligned}
$$

By the previous lemma, the first two components of the above inequality are finite, so it is enough to show that $I_{\varphi_{3}}\left(\lambda f \chi_{G_{1}} * g \chi_{G_{2}}\right)<\infty$. Since the support of $f \chi_{G_{1}} * g \chi_{G_{2}}$ is contained in $G_{1} G_{2}$

$I_{\varphi_{3}}\left(\lambda f_{G_{1}} * g \chi_{G_{2}}\right) \leqslant \int_{G_{1} G_{2} \varphi_{3}}\left(\lambda \int_{G_{1} \cap x G_{2}^{-1}}\left|f(t) \| g\left(t^{-1} x\right)\right| d \mu(t) d \mu(x)\right.$. There exists $u_{0} \geqslant 0$ such that

(8.2) $I_{\nabla_{1}}\left(6 \lambda / k f \chi_{\sigma_{1}}\right) \leqslant 1$ and $I_{\Phi_{2}}\left(g \chi_{c_{2}}\right) \leqslant 1$, where

$$
\tilde{G_{1}}=\left\{t \in G_{1}:|f(t)| \geqslant u_{0}\right\} \quad \tilde{G}_{2}=\left\{t \in G_{2}:|g(t)| \geqslant u_{0}\right\} .
$$

Denote $G_{0}=G_{1} \cap x G_{2}^{-1}$. We have

$$
\begin{gathered}
\lambda \int_{G_{0}-\tilde{G}_{1}}\left|f(t) \| g\left(t^{-1} x\right)\right| d \mu(t) \leqslant \lambda u_{0} \int_{G_{0}}\left|g\left(t^{-1} x\right)\right| d \mu(t) \\
=\lambda u_{0} \int_{G_{2} \cap \times \sigma_{1}}{ }^{-1}|g(u)| d \mu(u) \leqslant \lambda u_{0} \int_{G_{0}}|g(u)| d \mu(u)=M_{1}<\infty,
\end{gathered}
$$

since every function from Orlicz space is locally integrable. Analogously

$$
\lambda \int_{\sigma_{0} \mid x \sigma_{2}^{-1}}\left|f(t) \| g\left(t^{-1} x\right)\right| d \mu(t) \leqslant \lambda u_{0} \int_{\sigma_{1}}|f(t)| d \mu(t)=M_{2}<\infty .
$$

Thus,

$$
\begin{gathered}
I_{\mathrm{v}_{3}}\left(\lambda f f_{G_{1}} * g \chi_{G_{2}}\right) \leqslant \int_{G_{1} G_{2}} \varphi_{3}\left(M_{1}+M_{2}+\lambda \int_{G_{1} \cap \times G_{2}}|| f(t) \| g\left(t^{-1} x\right) \mid d \mu(t)\right) d \mu(x) \leqslant \\
1 / 3\left(\varphi_{3}\left(3 M_{1}\right)+\varphi_{3}\left(3 M_{2}\right) \mu G_{1} G_{2}+1 / 3 \int_{G_{1} G_{2}} \varphi_{3}\left(1 / 2 \int_{G_{1} \wedge x G_{2}^{-1}}\right.\right. \\
k 6 \lambda / k f f(t) \| g\left(t^{-1} x\right) d \mu(t) d \mu(x) .
\end{gathered}
$$

Denoting by $M_{3}$ the first component of the above sum and applying condition $(+)$ to the second one, we get

$$
\begin{gathered}
\mathrm{I}_{\varphi_{3}}\left(\lambda f \chi_{\sigma_{1}} * g \chi_{G_{2}}\right) \leqslant M_{3}+ \\
1 / 2 \int_{G_{1} G_{2}} \varphi_{3}\left(\int_{\sigma_{1} \cap x \sigma_{2}^{-1}} \varphi_{1}(6 \lambda / k f f(t))\right) \varphi_{3}^{-1}\left(\varphi_{2}\left(\mid g\left(t^{-1} x\right)\right)\right) d \mu(t) d \mu(x) \\
+1 / 2 \int_{G_{1} G_{2}} \varphi_{3}\left(\int_{\sigma_{1} \cap x \sigma_{2}}{ }^{-1} \varphi_{2}\left(\left|g\left(t^{-1} x\right)\right|\right) \varphi_{3}^{-1}\left(\varphi_{1}(6 \lambda / k f f(t) \mid)\right) d \mu(t) d \mu(x) .\right.
\end{gathered}
$$


In virtue of (8.2) and Jensen's inequality,

$$
\begin{aligned}
& I_{\varphi_{3}}\left(\lambda f \chi_{\sigma_{1}} * g \chi_{\sigma_{2}}\right) \leqslant M_{3}+ \\
& \left.1 / 2 \int_{G_{1} G_{2}}\left(\left(_{G_{1}} \cap x G_{2}^{-\mid} \varphi_{1}(6 \lambda / k f f t)\right)\right) \varphi_{2}\left(\left|g\left(t^{-1} x\right)\right|\right) d \mu(t)\right) d \mu(x)+ \\
& I / 2 \int_{\sigma_{1} \sigma_{2}}\left(\int_{\sigma_{1} \cap x G_{2}^{-1}} \varphi_{2}\left(\left|g\left(t^{-1} x\right)\right|\right) \varphi_{1}(6 \lambda / k f f(t)) d \mu(t) d \mu(x) \leqslant\right. \\
& M_{3}+I_{\varphi_{1}}\left(6 \lambda / k n I_{\omega_{2}}(g)<\infty .\right.
\end{aligned}
$$

If $G$ is compact and $\varphi$, satisfy, condition ( + ) for l.a., then by Lemma 5 there exist functions $\bar{\varphi}_{1}$ satisfing (+) for a.a. and such that $E_{\varphi_{i}}=E_{\dot{\theta}_{i}}, L_{\phi}=L_{\bar{\varphi}_{i}}$ So without loss of generality one can assume that $\varphi_{1}$ satisfy condition $(+)$ for a.a. Thus, the inclusions follow in the same way as above (we can put $G_{1}=G_{2} G$ ).

II. For this case, applying Lemma 5 again, we can also assume condition $(+)$ for a.a. In the inequality (8.1) we can see analogously as in I that the first two components are finite. The third component is also finite since the support of $f \chi_{\sigma_{1}}{ }^{*} g \chi_{c_{2}}$ is finite. So it is the end of the proof.

8. Lemma. If $\varphi_{1}$ satisfy condition (++) for s.a. then $l_{\varphi_{1}} \subset h_{\varphi_{3}}$ and $l_{\varphi_{2}} \subset h_{\varphi_{3}}$.

Proof. Using the equivalent form of condition $(++)$ expressed in Proposition 2 we have $\varphi_{1}^{-1}(u) \varphi_{2}^{-1}(u) \leqslant \alpha u \varphi_{3}^{-1}(k u)$ for $u \leqslant \delta$. But by concavity of $\varphi_{2}^{-1}$ there exists $\delta_{1}>0$ such that $u / \varphi_{2}^{-1}(u) \leqslant 1$ for $u \leqslant \delta_{1}$. So $\varphi_{1}^{-1}(u) \leqslant \alpha \varphi_{3}^{-1}(k u)$ for sufficiently small $u$. Putting $v=\varphi_{1}^{-1}(u)$ we obtain $\varphi_{3}(l / a v) \leqslant k \varphi_{1}(v)$ for small $v$, which immediately implies that

$\varlimsup_{u \rightarrow 0} \frac{\varphi_{3}(\lambda u)}{\varphi_{1}(u)}<\infty$ for all $\lambda>Q$. But the last condition implies the inclusion $l_{\phi_{1}} \leftrightarrow h_{\phi_{1}}([9])$.

\section{Theorem.}

1. Let $G$ be nondiscrete and $\varphi_{1}$ satisfy condition ( ++$)$ for l.a. if $G$ is compact and $(++)$ for a.a. if $G$ is noncompact. If $\varphi_{3}$ is finite, then $L_{\varphi_{1}}{ }^{*} L_{\varphi_{2}} \hookrightarrow E_{\varphi_{3}}$.

II. Let $G$ be discrete and $\varphi_{1}$ satisfy condition $(++)$ for s.a. Then $l_{\varphi_{1}}{ }^{*} l_{\varphi_{2}} \hookrightarrow h_{\varphi_{3}}$. 
Proof. I. Suppose $\mathrm{G}$ is compact. Take $f \in L_{q}, g \in L_{q}$ such that $I_{q}(f) \leqslant 1$ and $I_{\varphi,}(g) \leqslant 1$. Let $\lambda>0$ be arbitrary and $\delta>0$ be from condition (++) chosen for $\alpha=2 \lambda$. Put

$$
\begin{aligned}
& \left.G_{1}=\left\{t \in G: \varphi_{1}(\mid f(t))\right) \geqslant \delta\right\}, \\
& G_{2}=\left\{t \in G: \varphi_{2}(\mid g(t)) \geqslant \delta\right\} .
\end{aligned}
$$

The convolution $f * g$ is the sum of functions $f \chi_{G_{1}}{ }^{*} g \chi_{G_{2}}, f \chi_{G_{1}} * g \chi_{G G_{2}}$ and $f \chi_{G G_{1}} * g$. Applying (++) for 1.a. and Jensen's inequality, we get analogously as in the proof of Theorem 7 , that

$$
\begin{aligned}
& I_{\theta_{3}}\left(\lambda f \chi_{\sigma_{1}}{ }^{*} g \chi_{\sigma_{2}}\right) \leqslant \int_{\sigma} \varphi_{3}\left(\alpha / 2 \int_{\sigma} d f(t)\left|\chi_{\sigma_{1}}(t)\right| g\left(t^{-1} x\right) \mid \chi_{\alpha \sigma_{2}}{ }^{-1}(t) d \mu(t) d \mu(x) \leqslant\right. \\
& \int_{\sigma} \varphi_{3}\left[1 / 2 \int_{\sigma} \varphi_{1}\left(|f(t)| \chi_{\sigma_{1}}(t) \varphi_{3}^{-1}\left(k \varphi_{2}\left(\lg \left(t^{-1} x\right) \mid \chi_{\sigma \sigma_{2}^{-1}}(t)\right)\right) d \mu(t)\right.\right. \\
& +1 / 2 \int_{G} \varphi_{2}\left(\left|g\left(t^{-1} x\right)\right| \chi_{\alpha \sigma_{2}^{-1}}(t)\right) \varphi_{3}^{-1}\left(k \varphi_{1}\left(|f(t)| \chi_{\sigma_{1}}(t)\right)\right) d \mu(t) d \mu(x) \\
& \leqslant k I_{\bullet_{1}}(f) I_{\varphi_{2}}(g)<\infty \text {. }
\end{aligned}
$$

So it is enough to show that e.g. $I_{\varphi,}\left(\lambda f f_{G, G,} \neq g\right)<\infty$.

By local integrability of $g$, we have $M=\int d g(t) \mid d \mu(t)<\infty$.

Hence $I_{\varphi_{3}}\left(\lambda \chi_{\sigma_{G G_{1}}}{ } g\right) \leqslant \varphi_{3}\left(\chi_{\sigma_{1}^{-1}}(\delta) M\right) \mu G<\infty$.

In the case of noncompact $G$ the proof is similar and even simpler in the sense that $G_{1}=G_{2}=G$.

II. If $G$ is discrete and $f, g$, are the same as above, then there exist finite sets $G_{1}, G_{2} \subset G$ such that $I_{\theta_{1}}\left(\mathcal{X}_{\chi_{G G_{1}}}\right) \leqslant \delta$ and $I_{*}\left(g \mathrm{X}_{G G_{2}}\right) \leqslant \delta$, where $\delta>0$ is the constant from condition ( ++$)^{\prime}$ chosen for $\alpha=2 \lambda>0$. We have

$$
f * g=\left(f \chi_{\sigma_{1}} * g \chi_{\sigma_{2}}\right)+\left(f \chi_{G_{1}} * g \chi_{a G_{2}}\right)+\left(f \chi_{G G_{1}} * g \chi_{\sigma_{2}}\right)+\left(f \chi_{a G_{1}} * g \chi_{a G_{2}}\right) \text {. }
$$

The first component belongs to $h_{\phi}$ because its support is finite. Applying $(++)$ and Jensen's inequality to the last one similarly as in $I$, we get $I_{\mathrm{q}_{3}}\left(\lambda f_{\chi_{G G_{1}}}{ }^{*} g \mathrm{X}_{G G_{1}}\right) \leqslant k<\infty$.

To finish the proof note that

$$
\left|\left(f \chi_{G_{1}}{ }^{*} g \chi_{G G_{2}}\right)(x)\right| \leqslant \sum_{t \in G_{1}}\left|f(t) \| g\left(t^{-1} x\right)\right|
$$

for every $x \in G$, where $g\left(t^{-1}.\right) \in l_{\sigma_{2}}$ for all $t \in G$. Thus 


$$
\sum_{t \in \sigma_{1}}\left|f(t) \| g\left(l^{-1} .\right)\right| \in l_{\sigma_{2}} \text { and so } f \chi_{\sigma_{1}} * g \chi_{\sigma_{\sigma_{2}}} \in l_{\sigma_{2}} .
$$

But by Lemma 8, we have $l_{v_{2}} \subset h_{\varphi_{3}}$, so $f{\chi_{\sigma_{1}}}^{*} g \chi_{a G_{2}} \in h_{e_{3}}$.

Next theorems will be converse to the results obtained in Theorem 7 .

10. Theorem. Let $G$ be discrete. If $h_{\theta_{1}}{ }^{*} h_{\mathrm{q}_{2}} \subset l_{\sigma_{3}}$, then $G$ is finite or condition $(+)$ for s.a. is satisfied.

Proof. Remember, in the sequence case we have assumed the functions $\varphi_{i}$ have been finite. For a contrary, suppose the group $\mathrm{G}$ is infinite and condition $(+)$ for s.a. is not satisfied. There exist sequences $\left(u_{n}\right),\left(v_{n}\right)$ such that $\varphi_{1}\left(u_{n}\right) \rightarrow 0$ and $\varphi_{2}\left(v_{n}\right) \rightarrow 0$ and

$$
l / n u_{n} v_{n}>\varphi_{1}\left(u_{n}\right) \varphi_{3}^{-1}\left(\varphi_{2}\left(v_{n}\right)\right)+\varphi_{2}\left(v_{n}\right) \varphi_{3}^{-1}\left(\varphi_{1}\left(u_{n}\right)\right)
$$

Without loss of generality assume $\varphi_{1}\left(u_{n}\right) \geqslant \varphi_{2}\left(v_{n}\right)$.

We shall consider a few cases.

I. Let $\varphi_{1}\left(u_{n}\right) \geqslant \varphi_{2}\left(v_{n}\right)>0$, Let $\bar{u}_{n}$ be such that $\varphi_{1}\left(\bar{u}_{n}\right)=\varphi_{2}\left(v_{n}\right)$. Since $\varphi_{1}(u) / u$ is nondecreasing, $I / n \bar{u}_{n} \nu_{n}>\varphi_{1}\left(\bar{u}_{n}\right) \varphi_{3}^{-1}\left(\varphi_{2}\left(v_{n}\right)\right)$, by (10.1). So we can assume about $u_{n}, v_{n}$ that $\varphi_{1}\left(u_{n}\right)=\varphi_{2}\left(v_{n}\right)$ and

$$
1 / n u_{n} v_{n}>\varphi_{1}\left(u_{n}\right) \varphi_{3}^{-1}\left(\varphi_{2}\left(v_{n}\right)\right)
$$

We shall examine two types of the group $G$.

(a). Let $\mathrm{G}$ contain a cyclic subgroup of arbitrary large rank.

There exist natural numbers $l_{n}$ such that

$$
\left(2 l_{n}+1\right) \varphi_{1}\left(u_{n}\right) \leqslant 1 \text { and }\left(2 l_{n}+3\right) \varphi_{1}\left(u_{n}\right)>1 .
$$

Take a cyclic subgroup $S$ such that $r S>2 l_{n}+1$. Let

$$
A_{n}=\left\{t^{i} \in S: i=0,1, \ldots, l_{n},-1, \ldots,-l_{n}\right\},
$$

and

$$
f_{n}(t)=u_{n} \chi_{A_{n}}(t), g_{n}(t)=v_{n} \chi_{A_{n}}(t)
$$


By (10.3), it is evident that

$$
1 / 2 \leqslant I_{q_{1}}\left(f_{n}\right) \leqslant 1,1 / 2 \leqslant I_{q_{2}}\left(g_{n}\right) \leqslant 1 .
$$

Moreover

$$
\left(f_{n}^{*} g_{n}\right)(x)=u_{n} v_{n} \mu\left(A_{n} \cap A_{n} x\right)
$$

If $x \in A_{n}$ then either $A_{n} \cap A_{n} x \supset\left\{e, t, \ldots, t^{\prime}\right\}$ or $A_{n} \cap A_{n} x \supset\left\{e, t^{-1}, \ldots, t^{-t_{n}}\right\}$. Therefore $\mu\left(A_{n} \cap A_{n} x\right) \geqslant l_{n}+l$ for $x \in A_{n}$.

Hence

$$
\left(f_{n}^{*} g_{n}\right)(x) \geqslant u_{n} v_{n}\left(l_{n}+I\right) \chi_{n}(x)
$$

But by (10.3), we get the following estimation of $l_{n}$

$$
l_{n}+1 \geqslant I / 3 \varphi_{1}\left(u_{n}\right)
$$

Thus in virtue of (10.2)

$$
\frac{3}{n}\left(f_{n} * g_{n}\right)(x) \geqslant \frac{I}{n} u_{n} v_{n} \frac{l}{\varphi_{1}\left(u_{n}\right)} \chi_{A_{n}}(x)>\varphi_{3}^{-1}\left(\varphi_{2}\left(v_{n}\right)\right) \chi_{A_{n}}(x) .
$$

Hence and by Lemma 1 and the fact that $\varphi_{3}\left(b_{3}\right)=\infty$ and by (10.4), we have

$$
I_{\varphi_{3}}\left(3 / n f_{n} * g_{n}\right) \geqslant \varphi_{2}\left(v_{n}\right) \mu A_{n}=I_{\varphi_{2}}\left(g_{n}\right) \geqslant 1 / 2 .
$$

So we found sequences $f_{n} \in h_{\varphi_{1}}, g_{n} \in \mathrm{h}_{\phi_{2}}$ such that $I_{\theta_{1}}\left(f_{n}\right) \leqslant 1, I_{\varphi_{2}}\left(g_{n}\right) \leqslant 1$ and $I_{\omega_{3}}\left(3 / n f_{n} * g_{n}\right) \geqslant 1 / 2$. Applying Theorem 1.2 we can see that $h_{\phi_{1}} * h_{q_{2}} \notin l_{\varphi_{3}}$

(b). Let, contrary to (a), the rank of all cyclic subgroups of $G$ be bounded. So there exists a prime number $k$ and infinite number of cyclic subgroups with rank equal to $k$. Let $S_{i}$ be an infinite sequence of cyclic subgroups such that $r S_{i}=k, S_{i} \cap S_{j}=\{e\}$ for $i=j$. Let $P_{n}=\underset{i=1}{\oplus} S_{i}$ be a simple sum of $S_{1}, \ldots, S_{n^{*}}$. The set $P_{n}$ is a subgroup of $G$ containing $k^{n}$ different elements. Put

$$
\begin{gathered}
l_{n}=\left[\log _{k} \frac{1}{\varphi_{1}\left(u_{n}\right)}\right] \text { and } \\
f_{n}(t)=u_{n} \chi_{P_{l_{n}}}(t) \text { and } g_{n}(t)=v_{n} \chi_{P_{l_{n}}}(t) .
\end{gathered}
$$

It is clear that 


$$
1 / k \leqslant I_{\varphi_{1}}\left(f_{n}\right) \leqslant 1,1 / k \leqslant I_{\oplus_{2}}\left(g_{n}\right) \leqslant 1 .
$$

Moreover,

$$
\left(f_{n}^{*} g_{n}\right)(x) \geqslant u_{n} v_{n} \mu P_{l_{n}} \chi_{P_{n}}(x)=u_{n} v_{n} k^{l} \chi_{P_{n}}(x) .
$$

But by the definition of $l_{n}$, we have

$$
k^{\prime} \geqslant 1 / k \varphi_{1}\left(u_{n}\right) .
$$

Thus in virtue of (10.2), Lemma 1 and (10.7) we obtain

$$
I_{\nabla_{3}}\left(k / n f_{n} * g_{n}\right) \geqslant I_{q_{2}}\left(g_{n}\right) \geqslant 1 / k .
$$

So applying Theorem 1.2 we get a contradiction.

II. Suppose $\varphi_{1}\left(u_{n}\right)>\varphi_{2}\left(v_{n}\right)=0$. Hence $a_{2}>0$. We shall consider two cases. Let first $a_{3}=0$. There exists an infinite, countable subgroup $G_{o}$ of $G$. There exists an element $\left(c_{n}\right) \in \mathcal{c}_{o}$ such that $\left(c_{n}\right) \notin l_{\mathrm{\varphi}_{3}}\left(G_{o}\right)$. Put

$$
\begin{aligned}
& u(t)=\left\{\begin{array}{r}
c_{n} \text { if } t=t_{n}, n=0,1,2, \ldots \\
0 \text { if } t \notin G_{o}
\end{array}\right. \\
& v(t)=\chi_{|e|}(t),
\end{aligned}
$$

where $G_{e}=\left\{e, t_{1}, t_{2}, \ldots,\right\}$. Since $a_{2}>0, h_{e_{2}}$ is isomorphic to $c_{e^{*}}$ So it is clear that $u \in h_{\phi_{2}}, v \in h_{\phi_{1}}$ and $u^{*} v=\notin l_{q_{3}}$.

Now let $a_{3}>0$. In this case we modify the proof of the part I. Let $C_{n}$ denote $A_{n}$ or $P_{i}$ and $c_{n}$ denote $l_{n}$ or $k^{\prime}$ and $c$ be equal to 3 or $k$, respectively, when $G$ satisfies $(a)$ or $(b)$. Let $f_{n}(t)=u_{n} \chi_{C_{n}}(t)$ and $g_{n}(t)=v_{n} \chi_{c_{n}}(t)$. Then $\min (1 / 2$, $1 / k) \leqslant I_{\varphi_{1}}\left(f_{n}\right) \leqslant 1, I_{\Phi_{2}}\left(g_{n}\right) \leqslant 1$ and

$$
\left(f_{n}^{*} g_{n}\right)(x) \geqslant u_{n} v_{n} \frac{I}{c \varphi_{1}\left(u_{n}\right)} \chi_{c_{n}}(x),
$$

by $(10.4),(10.7),(10.5),(10.6)$ and $(10.8)$. Hence and by (10.1) we get

$$
\begin{aligned}
& I_{\varphi_{3}}\left(2 c / n f_{n}^{*} g_{n}\right) \geqslant \int_{G} \varphi_{3}\left(2 \varphi_{3}^{-1}\left(\varphi_{2}\left(\nu_{n}\right)\right)\right) \chi_{c_{n}}(x) d \mu(x) \\
= & \int_{\sigma} \varphi_{3}\left(2 a_{3}\right) \chi_{c_{n}}(x) d \mu(x)=\varphi_{3}\left(2 a_{3}\right) \mu C_{n} \rightarrow \infty, \text { as } n \rightarrow \infty .
\end{aligned}
$$

So by virtue of Theorem 1.2 and the fact that $f_{n} \in h_{\varphi_{1}}$ and $g_{n} \in h_{\vartheta_{2}}$ we get the hypothesis. 
III. If $\varphi_{1}\left(u_{n}\right)=\varphi_{2}\left(v_{n}\right)=0$ then $a_{i}>0$ for $i=1,2$. So $h_{0}\left(G_{o}\right)$ is isomorphic to $c_{o}$ for $i=1,2$ and any infinite, countable subgroup $G_{o}$ of ${ }^{i} G$. But it is possible to construct elements $u, v \in c_{o}$ such that $\left(u^{*} v\right)(e)=\infty$.

11. Theorem. For any noncompact abelian group $G$ condition $(+)$ for s.a. is necessary for the inclusion $L_{q_{1}} * L_{q_{2}} \subset L_{\boldsymbol{q}_{3}}$. If additionally $\varphi_{i}(i=1,2)$ are finite then condition (+) for s.a. is necessary for the inclusion $E_{\boldsymbol{q}_{1}} * E_{\mathrm{q}_{2}} \subset L_{\boldsymbol{\varphi}_{3}}$, too.

Proof. By the well known results about the structure of an abelian group either the group $G$ contains a compact open subgroup $G_{o}$ or it contains an element $z$ such that the set $\left\{z^{n}: n \in \mathbb{Z}\right\}$ is an infinite, discrete subgroup of $G$, where $\mathbb{Z}$ is the set of all integers. Let $\tilde{\varphi}_{i}$ be finite and equivalent to $\varphi_{i}$ for s.a. (see Lemma 4).

In the first case $G / G_{o}$ is infinite and discrete. Suppose $\mu G_{o}=1$. We shall show that $l_{\theta_{1}}\left(G / G_{o}\right) * l_{\theta_{2}}\left(G / G_{o}\right) \subset l_{\sigma_{3}}\left(G / G_{o}\right)$. For $f \in l_{\phi_{1}}\left(G / G_{o}\right)$ and $g \in l_{\phi_{0}}\left(G / G_{o}\right)$ put $\tilde{f}(x)=f\left(x G_{o}\right)$ and $\bar{g}(x)=g\left(x G_{o}\right)$ where $x G_{o}$ belongs to $G / G_{o}$. Then $f^{2} \in l_{\varphi}\left(G / G_{o}\right)$, $g \in l_{q}\left(G / G_{o}\right)$ and clearly $\bar{f} \in L_{q}$ and $\bar{g} \in L_{q}$. By the assumption $\bar{f} \bar{g} \in L_{\mathrm{q}}$. But $f * \overline{\mathrm{g}}(x)=f * \mathrm{~g}\left(x G_{0}\right)$ for all $x \in G$, because $\mu G_{o}=1$. Therefore $f * g \in l_{q_{3}}\left(G / G_{o}\right)=l_{\phi_{3}}\left(G / G_{o}\right)$. Thus by virtue of finiteness of $\tilde{\varphi}_{i}$ and Theorem 10 , $\bar{\varphi}_{i}$ satisfy condition $(+)$ for $\mathbf{s} . \mathbf{a}$. But Proposition 3 implies that $\varphi_{i}$ satisfy condition (+) for s.a., too.

In the second case denote $G_{1}=\left\{z^{n}: n \in \mathbb{Z}\right\}$. Analogously as above it is enough to show that $l_{\phi_{1}}\left(G_{1}\right) * l_{\phi_{1}}\left(G_{1}\right) \subset l_{\phi_{1}}\left(G_{1}\right)$. Take arbitrary $a=\left(a_{n}\right) \in l_{\phi_{0}}\left(G_{1}\right)=l_{\Phi_{1}}\left(G_{1}\right)$ and $b=\left(b_{n}\right) \in l_{\phi_{2}}\left(G_{1}\right)=l_{\Phi_{2}}\left(G_{1}\right)$. Then $a * b=c=\left(c_{n}\right)$ where $c_{n}=\sum_{k \in \mathbf{Z}} a_{k} b_{n-k}$

Let $U, V$ be symmetric neighbourhoods of $e$ such that

$U \cap G_{1}=\{e\}$ and $V^{2} \subset U$. Put $f(t)=\sum_{n \in Z} a_{n} \chi_{U_{z}^{n}}(t), g(t)=\sum_{n \in \mathbb{Z}} b_{n} \chi_{U z^{n}}(t)$. Clearly $f \in L_{\varphi_{1}}$, $g \in L_{\varphi_{2}}$ and so $f * g \in L_{\varphi_{3}}$. Moreover,

$$
\left.|f|^{*}|g|(x) \geqslant \sum_{n \in \mathbf{Z}}\left|a_{k}\right| b_{n-k}\right\rfloor \mu\left(U x z^{-n} \cap U\right) .
$$

If $x \in V z^{n}$ then $x z^{-n} V \subset: V^{2} \subset U$. Hence $\mu\left(U x z^{-n} \cap U\right) \geqslant \mu V$.

Thus $|f| *|g|(x) \chi_{V z^{n}}\left(x|\geqslant| c_{n} \mid \mu V\right.$. Therefore there exists $\lambda>0$ such that $\sum_{n \in Z} \varphi_{3}\left(\lambda_{\mu}\left|c_{n}\right|\right) \leqslant I_{\phi_{3}}(\lambda j|||| g \mid)<\infty$, which shows that $c=\left(c_{n}\right) \in l_{\phi_{3}}\left(G_{1}\right)=l_{q_{3}}\left(G_{1}\right)$. Thus the first part of he theorem is proved. The proof of the second one is similar and even simp'er because the functions $\varphi_{i}$ are finite by the assumption. 
Now, le us introduce two conditions for a locally compact group.

We say that a group $G$ satisfies condition (*) if for every sequence $\alpha_{t} \rightarrow \infty$ there exist sequences $\left(U_{i}\right),\left(V_{i}\right)$ of measurable sets and constants $\kappa, k_{1}, k_{2}>0$ such that

- $k_{1} \leqslant \alpha_{i} \mu U_{i} \leqslant k_{2}$,

$-V_{i} V_{i}^{-1} \subset U_{p}$

$-\mu U_{i} \leqslant \kappa \mu V_{i}$

for every $i \in \mathbb{N}$.

It is said that a sequence $\left(U_{i}, V_{i}\right)$ is a so called $\mathrm{D}$ "-sequence ([2]) if $U_{i}, V_{i}$ are measurable sets and there exists $\mathrm{K}>0$ such that

- $U_{1} \supset U_{2} \supset \ldots, U_{i} \rightarrow e_{\text {, }}$

$-V_{i} V_{i}^{-1} \subset U_{i}$

$-\mu U_{j} \leqslant \kappa \mu V_{i}$,

for every $i \in \mathbb{N}$.

Note, in the above two conditions we may always suppose that $V_{i} \subset U_{i}$.

12. Remark. The following groups satisfy condition $\left({ }^{*}\right)$ : $(\mathbb{R},+),(\mathbf{K},+),(T, \cdot),(\mathbb{R} \backslash\{O\}, \cdot),(\mathbf{K} \backslash\{0\}, \cdot)$, where $\mathrm{T}$ is a subgroup of $(\mathbf{K} \backslash\{O\},$.$) con-$ sisting of all elements belonging to the unit sphere of $\mathbb{K}$.

In [2] there are examples of groups addmiting a D"-sequence. For instance the groups containing an open subgroup of the form $\mathbb{R}^{a} \times T^{3} \times F$, where $a, b$ are positive integers and $F$ is a finite group, admit a $D$ "-sequence.

13. Proposition. If a group $G$ contains an infinite, discrete and cyclic subgroup and $G$ admits a $D^{\prime \prime}$-sequence, then the condition $\left(^{*}\right)$ is satisfied.

Proof. Let $\left(\alpha_{i}\right), \alpha_{i} \geqslant 1$, be an arbitrary sequence tending to infinity and $\left\{z^{n}: n \in \mathbb{Z}\right\}$ be a discrete subgroup of $G$. If $W$ is neighbourhood of $e$ such that $\left\{z^{n} W\right\}$ is a pairwise disjoint family of sets, then we may assume that $U_{i} \subset W$, 


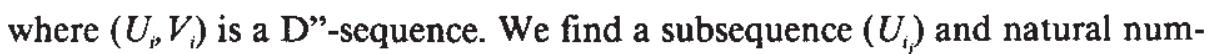
bers $k_{j}$ such that $I / 2 \leqslant \alpha_{j}\left(2 k_{i}+I\right) \mu U_{i j} \leqslant 1$. Putting

$P_{j}=\bigcup_{n=-2 k_{j}}^{2 k_{j}} z^{n} U_{i_{j}}, Q_{j}=\bigcup_{n=-k_{j}}^{k_{j}} z^{n} V_{i_{j}}$, we have

$\mu P_{j}=\left(4 k_{j}+1\right) \mu U_{i_{j}}$ and $Q_{j}=\left(2 k_{j}+1\right) \mu V_{i j}$. Hence

$I / 4 \leqslant \alpha_{j} \mu P_{j} \leqslant 2$ and $\mu P_{j} \leqslant 2 \kappa \mu Q_{j}$. Moreover,

$$
\begin{aligned}
Q_{j} Q_{j}^{-1} & =\bigcup_{n=-k_{j} m=-k_{j}}^{k_{j}} z^{k_{j}} \bigcup_{i=m}^{k_{j}} V_{i j}^{-1} V_{i_{j}}=\bigcup_{i=-2 k_{j}}^{2 k_{j}} z^{i} V_{i_{j}}^{-1} V_{i_{j}} \\
& \subset \bigcup_{i=-2 k_{j}}^{2 k_{j}} z^{i} U_{i_{j}}=P_{f}
\end{aligned}
$$

Thus the group $G$ satisfies condition $\left({ }^{*}\right)$.

14. Theorem. Let a group $G$ satisfy condition $\left({ }^{*}\right)$. Then condition $(+)$ for l.a. is necessary for the inclusion $L_{\boldsymbol{\varphi}_{1}} * L_{\boldsymbol{\varphi}_{2}} \subset L_{\boldsymbol{\varphi}_{3}}$.

If moreover $\varphi_{i}(i=1,2)$ are finite, then the condition ( +$)$ for l.a. is necessary for the inclusion $E_{\boldsymbol{q}_{1}} * E_{\mathrm{q}_{2}} \subset L_{\boldsymbol{\theta}_{3}}$, too.

Proof. Assume $\varphi_{i}(i=1,2)$ are finite (in another case condition ( + ) for l.a. is always satisfied). Suppose condition ( + ) for l,a. is not sastified. Then there exist sequences $\left(u_{i}\right),\left(v_{i}\right)$ such that $\varphi_{1}\left(u_{i}\right) \rightarrow \infty$ and $\varphi_{2}\left(v_{i}\right) \rightarrow \infty$ and

$$
\left.1 / i u_{i} v_{i}>\varphi_{1}\left(u_{i}\right) \varphi_{3}^{-1}\left(\varphi_{2}\left(v_{i}\right)\right)+\varphi_{2}\left(v_{i}\right) \varphi_{3}^{-1}\left(\varphi, u_{i}\right)\right) .
$$

Analogously as in the proof of Theorem 10 one can put $\varphi_{1}\left(u_{i}\right)=\varphi_{2}\left(v_{i}\right)$. By the assumed condition $\left({ }^{*}\right)$, one can find a sequence $U_{i}$ of measurable sets such that

$$
k_{1} \leqslant \varphi_{1}\left(u_{i}\right) \mu U_{i} \leqslant k_{2}
$$

where $k_{1}, k_{2}>0$. Putting

$$
f_{i}(t)=u_{i} \chi_{U_{i}}(t), g_{i}(t)=v_{i} \chi_{U_{i}^{-1}}(t),
$$

we have

$$
f_{i}^{*} g_{i}(x)=u_{i} v_{i} \mu\left(x U_{i} \cap U_{j}\right) .
$$

We can assume that $V_{i} \subset U_{i}$, where $V_{i}$ are sets from condition (*). So, if $x \in V_{i}^{-1}$ then $x V_{i} \subset U_{i}$ and 


$$
\mu\left(x U_{1} \cap U_{1}\right) \geqslant \mu\left(x U_{i} \cap x V_{j}\right)=\mu V_{b}
$$

for $x \in V_{1}^{-1}$. Thus

$$
f_{i} \star g_{i}(x) \geqslant u_{i} v_{\mu} \mu V \chi_{r_{i}}{ }^{\prime}(x)
$$

Then in virtue of (14.1) and (14.2), we get

$$
\kappa / k_{1} l / i\left(f_{i}^{*} g_{3}\right)(x) \geqslant \kappa / k_{1} \varphi_{1}\left(u_{i}\right) \varphi_{3}^{-1}\left(\varphi_{2}\left(v_{i}\right)\right) \mu V_{i} \chi_{r_{i}^{-1}}(x) \geqslant \varphi_{3}^{-1}\left(\varphi_{2}\left(v_{1}\right)\right) \chi_{r_{t}^{-1}}(x) .
$$

Therefore

$$
I_{\varphi_{j}}\left(\kappa / k_{1} \quad l / i f_{i}^{*} g_{1}\right) \geqslant I / \kappa \varphi_{3}\left[\varphi_{3}^{-1}\left(\varphi_{2}\left(v_{i}\right)\right)\right] \mu U_{r}
$$

Now if $b_{3}=\infty$ then

$$
I_{\mathrm{e}_{3}}\left(\kappa / k_{1} l / i f_{1} * g_{1}\right) \geqslant k_{1} / \mathrm{\kappa} \text { for every } i \in \mathbb{N},
$$

if $b_{3}<\infty$ then $4 \varphi_{3}^{-1}\left(\varphi_{2}\left(v_{i}\right)\right)>2 b_{3}$ for sufficiently large $i$ and so

$$
I_{\varphi_{3}}\left(\frac{4 \kappa}{\kappa_{1}} \frac{l}{i} f_{i} * g_{3}\right) \geqslant \varphi_{3}\left(2 b_{3}\right) \mu V_{i}=\infty .
$$

Thus we have found sequences $\left(f_{i},\left(g_{i}\right)\right.$ such that $f_{i} \in E_{q_{1}}, g_{i} \in E_{t_{2}}$ and $I_{i_{1}}(D) \leqslant k_{2}$, $I_{\sigma_{2}}\left(g_{i}\right) \leqslant k_{2}$ and $I_{\theta_{3}}\left(\lambda_{i} f_{i} * g_{i}\right) \geqslant$ const. for some $\lambda_{1} \rightarrow 0$. Then, by Theorem 1. The inclusions $E_{\phi_{1}} * E_{\varphi_{2}} \subset L_{\varphi_{3}}$ and $L_{\varphi_{1}}{ }^{*} L_{\varphi_{2}} \subset L_{\varphi_{3}}$ are not fulfilled, which ends the proof of the theorem.

The following three corollaries are immediate consequence of Theorems $1.1,1.2,2,7,10,11$ and 14 .

15. Corollary. Let $G$ be a discrete group. The following conditions are equivalent
(1) $l_{\varphi_{1}} * l_{\sigma_{2}} \hookrightarrow l_{\omega_{3}}$
(2) $h_{\varphi_{1}} * l_{\psi_{2}} \hookrightarrow h_{\varphi_{3}}$
(3) $h_{\varphi_{1}} * l_{\phi_{2}} c l_{\varphi_{1}}$
(4) $h_{\varphi_{1}} l_{\varphi_{2}} \leftrightarrow l_{\phi_{3}}$
(5) $\varphi$ satisfies condition $(+)$ for s.a. or $G$ is finite, 
(6) There exist $l, \delta>0$ such that

$$
\varphi_{1}^{-1}(u) \varphi_{2}^{-1}(u) \leqslant l u \varphi_{3}^{-1}(u)
$$

if $u \leqslant \delta$, or $G$ is finite,

(7) $\|f * g\|_{\bullet_{3}} \leqslant c\|f\|_{\bullet_{1}}\|g\|_{\bullet_{2}}$

$$
\text { for some } c>0 \text { and all } f \in l_{l_{1}}, g \in l_{\sigma_{2}} \text {. }
$$

16. Corollary. Let $G$ be nondiscrete group and $\varphi_{i}(i=1,2,3)$ be finite. Consider the conditions (1) to (4) and (7) as in Corollary 15, where $l_{\vartheta_{i}} h_{\bullet_{i}}$ are replaced by $L_{q_{i}} E_{q_{i}}$ respectively. Moreover, let

(5) $\varphi$ satisfies condition (+) for l.a. if $G$ is compact or for a.a. if $G$ is noncompact,

(6) there exist l, $\delta>0$ such that

$$
\varphi_{1}^{-1}(u) \varphi_{2}^{-1}(u) \leqslant \operatorname{lu} \varphi_{3}^{-1}(u)
$$

if $u \geqslant \delta$ and $G$ is compact or if $u \geqslant 0$ and $G$ is noncompact.

We have relations: $(I) \leftrightarrow(7)$ and

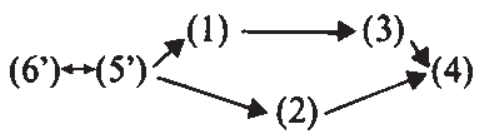

Moreover, if a group $G$ satisfies condition (*) then $(4) \rightarrow(5)$, i.e. all the above conditions are equivalent. [8]).

Sufficiency of the next corollary is known as Young theorem (see e.g. [2],

17. Corollary. Let $l \leqslant p, q, r<\infty$.

I. If $G$ is discrete and infinite, then

$$
P * k \bullet l^{r}
$$

$$
\text { if } 1 / p+1 / q \geqslant 1 / r+1 \text {. }
$$


II. Let $G$ be nondiscrete compact and $1 / p+1 / q \leqslant 1 / r+1$ or respectively $G$ is noncompat and $1 / p+1 / q=1 / r+1$, then

$$
L^{p *} L^{q \hookrightarrow} \rightarrow L
$$

If additionally $\mathrm{G}$ satisfies condition (*), then the converse of the above is also true.

\section{Corollary.}

$I$ (th. 2 in [3]) $L_{\mathrm{o}}$ is a Banach algebra under convolution as multiplication iff $L_{\bullet} \hookrightarrow L^{1}$, i.e. $\lim _{u \rightarrow 0} \varphi(u) / u>0$ or $G$ is compact.

II ([10]) $L^{p}(I \leqslant p<\infty)$ is a Banach algebra iff $p=1$ or $G$ is compact.

Proof. I. If we put $\varphi_{i}=\varphi(i=1,2,3)$, then $\varphi_{i}$ satisfy condition (+) for l.a., by convexity of $\varphi$. Moreover, if $\mathrm{L}_{\varphi}$ is a Banach algebra and $\mathrm{G}$ is noncompact, then applying Theorem 11 we get condition $(+)$ for s.a. Thus $\varphi_{i}$ satisfy $(+)$ for a.a., which means that $\lim \varphi(u) / u>0$. The converse is immediate, by Theorem 7.

The point I of the above Corollary (see also [3]) is the answer to the Gramsch's problem from [1], in the case of convex function $\Phi$.

\section{References}

[1] B. GramsCh, Die Klasse metrischer linearer Raume, Math Annalen 171, 61-78 (1967).

[2] E HEwITT and K.A. Ross, Abstract Harmonic analysis I, II, SprngerVerlag 1963-1970.

[3] H. HudziK, A. KAMIŃSKA and J. MuSIElaK, On some Banach algebras given by a modular, Colloq. Math. Soc. J. Bolyai, Alfred Haar Memorial Conference, Budapest 49, 445-463 (1985).

[4] M.A. KRASNOSELSKII and YA.B. RUTICKII, Convex functions and Orlicz spaces, Groningen 1961.

[5] W.A.J. LUXEMBURG, Banach function spaces, thesis 1955.

[6] W.A.J. LuXemburg and A.C. ZAANEN, Riesz spaces, I, North Holland 1971.

[7] J. MUSIELAK, Orlicz spaces and modular spaces, Lect. Notes in Math. 1034, Springer-Verlag 1983. 
[8] R.O'NEIL, Fractional integration in Orlicz spaces I, Trans. of A.M.S. 115 , 300-329 (1965).

[9] M. NowaK, On inclusions between Orlicz spaces, Comment. Math. 26 (1968).

[10] W. ZELAZKo, On the algebras $L$ of locally compact groups, Colloq. Math. 8, 115-120 (1961).

INSTITUTE OF MATHEMATICS

A. MICKIEWICZ UNIVERSITY

POZNAN, POLAND 